\title{
Albumin and Antioxidants Inhibit Serum-deprivation-induced Cell Adhesion in Hematopoietic Cells
}

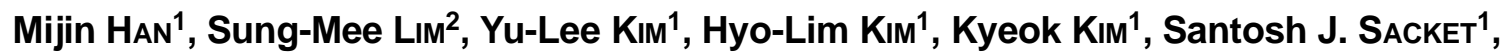 \\ Ji-Yeong Jo', Yoe-Sik BAE ${ }^{3}$, Fumikazu OKAJIMA ${ }^{4}$, and Dong-Soon $\mathrm{Im}^{1 *}$ \\ ${ }^{1}$ Laboratory of Pharmacology, College of Pharmacy (BK21 Project) and Research Institute for Drug Development, \\ Pusan National University, Busan 609-735, Republic of Korea \\ ${ }^{2}$ Department of Food Science \& Technology, Tongmyong University, Busan 608-711, Republic of Korea \\ ${ }^{3}$ Department of Biochemistry, College of Medicine, Dong-A University, Busan 602-714, Republic of Korea \\ ${ }^{4}$ Laboratory of Signal Transduction, Institute for Molecular and Cellular Regulation, Gunma University, \\ Maebashi, Gunma 371, Republic of Japan
}

(Received May 16, 2008; Revised May 27, 2008 ; Accepted June 10, 2008)

\begin{abstract}
Previously, we identified albumin as an inhibitory factor in serum for cell adhesion of $T$ cells such as human Jurkat $T$ and primary cultured human $T$ cells. In the present study, we found that other hematopoietic cell lines including U-937 human monocytes, THP-1 human monocytes, K-562 promyelocytic leukemia cells, and HL-60 human leukemia cells, also adhere to tissue culture flasks when serum is withdrawn, and albumin exerts an inhibitory effect on cell adhesion by those cells, implying that this inhibition is a common phenomenon in hematopoietic cells. Furthermore, we found that cell adhesion is inhibited by antioxidants such as (-)-epigallocatechin-3-gallate (EGCG), morin, and a-tocopherol. Our results suggest that albumin may inhibit basal cell adhesion of hematopoietic cells and that the oxidative balance in the plasma may be important for cell adhesion of hematopoietic cells in vivo.
\end{abstract}

Keywords : Monocytes, Leukocytes, Albumin, Adhesion, Hematopoietic cells, Oxidative signaling

Abbreviations - BSA, bovine serum albumin; FBS, fetal bovine serum; EGCG, (-)-epigallocatechin-3-gallate; morin, 3,5,7,2',4'-pentahydroxyflavone; ECM, extracellular matrix

\section{INTRODUCTION}

Hematopoietic cells including $T$ cells and monocytes circulate in the blood. When they are activated by a variety of stimuli such as cytokines, they migrate to target tissues where immune responses occur (Dustin and de Fougerolles, 2001; Tseng and Dustin, 2002). The attachment and migration of activated cells are well characterized and the involvement of adhesion molecules such as ICAMs has been investigated extensively (Campanero et al., 1994; Dustin and de Fougerolles, 2001; Tseng and Dustin, 2002). However, cell adhesion and migration of inactivated hematopoietic cells has been poorly investigated. In the previous study, we reported that bovine serum albumin (BSA), the

${ }^{*}$ Corresponding author

Tel: +82-51-510-2817, Fax: +82-51-513-6754

E-mail: imds@pusan.ac.kr most studied and well-understood protein in plasma, inhibited the adhesion of $T$ cells, such as the human Jurkat $T$ cell line and primary cultured human T cells, probably independent of integrin-mediated signaling (Kim et al., 2006). In the present study, we tested whether this effect of albumin is common in hematopoietic cells by using U-937 human monocytes, THP-1 monocytes, K-562 promyelocytic leukemia cells, and HL-60 human leukemia cells and also whether oxidative signaling is involved in cell adhesion by using various antioxidants such as (-)-epigallocatechin-3-gallate (EGCG), morin, and $\alpha$-tocopherol, because oxidative signaling has been implicated in inflammation-mediated cell adhesion (Chen et al., 2006; Sen and Roy, 2001).

\section{METHODS AND MATERIALS}

\section{Materials and cell culture}

Fetal bovine serum (FBS) was purchased from Hyclone 

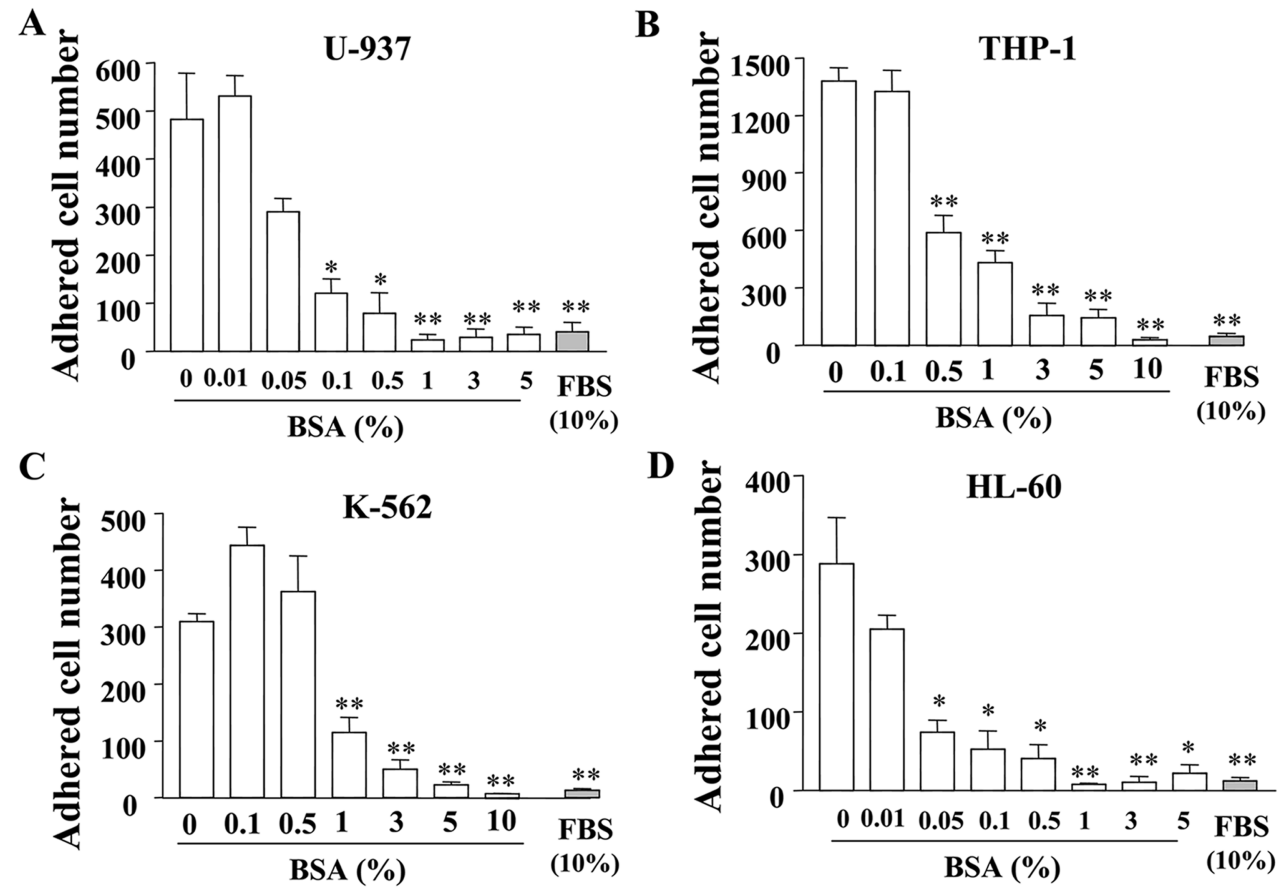

Fig. 1. Inhibitory effect of albumin on cell adhesion of hematopoietic cells BSA concentration-dependence of cell adhesion in U-937 (A), THP-1 (B), K-562 (C), and HL-60 cells (D). Cells were incubated for 30 min in tissue culture dishes with 10\% FBS (filled column) or without FBS and various concentrations of BSA (open column), and the number of adherent cells was calculated. Statistical significance: * $P<0.05,{ }^{* *} P<0.01$ vs. control without FBS and BSA.

(Logan, UT, USA) and bovine serum albumin (BSA, essentially fatty acid-free), EGCG, and $\alpha$-tocopherol were from Sigma (St. Louis, MO, USA). Morin (3,5,7,2',4'-pentahydroxyflavone) was provided by the Aging Tissue Bank, Research Institute of Genetic Engineering (Pusan National University). Jurkat T cells, U-937 monocytes (CRL-1593.2), THP-1 monocytes (TIB-202), HL-60 human leukemia (CCL-240), and K-562 promyelocytic leukemia cells (CCL243) were purchased from American Type Culture Collection (Manassas, VA, USA) and maintained in RPMI 1640 media, containing $10 \%(\mathrm{v} / \mathrm{v})$ FBS, 100 units/ml penicillin, $50 \mu \mathrm{g} / \mathrm{ml}$ streptomycin, $2 \mathrm{mM}$ glutamine, and $1 \mathrm{mM}$ sodium pyruvate at $37^{\circ} \mathrm{C}$ in a humidified $5 \% \mathrm{CO}_{2}$ incubator.

\section{Cell adhesion}

Cells $\left(5 \times 10^{5} \mathrm{cell} / \mathrm{sL}\right)$ were seeded onto 48 -well plates (BD Falcon, San Jose, CA, USA) for $30 \mathrm{~min}$ at $37^{\circ} \mathrm{C}$. Media were discarded and non-adherent cells were removed by washing the wells twice with phosphate-buffered saline (PBS). Adherent cells were counted or stained with eosin Y. For eosin $Y$ staining, cells that adhered to the wells were fixed overnight with $2 \%$ formaldehyde and stained with eosin $Y$ for 30 min. Eosin $Y$ was then extracted by the addition of a mixture of $1 \%$ of acetic acid and $50 \%$ ethanol, and the absorbance was measured at $570 \mathrm{~nm}$ (Kim et al., 2006).

\section{Statistical analysis}

The results are expressed as mean \pm SE of the number of determinations indicated. Statistical significance of differences was determined by ANOVA. Significance was accepted when $\mathrm{P}<0.05$.

\section{RESULTS}

\section{Inhibitory effect of albumin on adhesion of hemato- poietic cells}

Jurkat $\mathrm{T}$ cells or human primary $\mathrm{T}$ cells adhered to a tissue culture flask when $10 \%$ fetal bovine serum (FBS) was withdrawn (Kim et al., 2006), and T cell adhesion was inhibited by bovine serum albumin (BSA) in FBS (Kim et al., 2006). To test whether this effect of albumin is common in hematopoietic cells, we investigated the effects of FBS and albumin on cell adhesion in U-937 monocytes, THP-1 monocytes, K-562 promyelocytic leukemia cells, and HL-60 human leukemia cells. As shown in Fig. 1, we found that the cells adhered to a tissue culture flask when $10 \%$ FBS was withdrawn and that albumin inhibited the adhesion of all tested cell lines in a concentration-depen- 

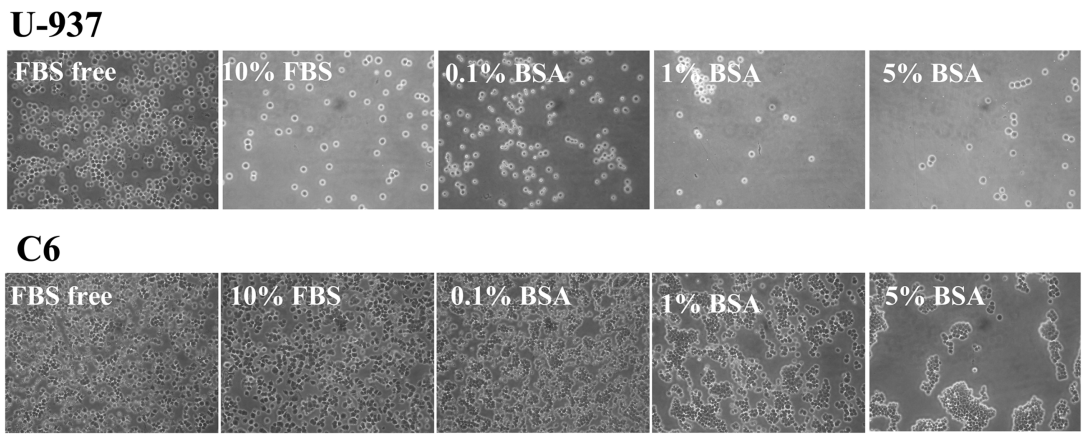

Fig. 2. Effects of FBS and BSA on cell adhesion of U-937 monocytes and C6 glioma cells U-937 monocytes (upper panels) or C6 glioma cells (lower panels) were incubated for $30 \mathrm{~min}$ for U-937 cells or $1 \mathrm{~h}$ for $\mathrm{C} 6$ cells in tissue culture dishes without FBS, with $10 \%$ FBS or with various concentrations of BSA in the culture media. Cells were photographed. Phase-contrast photographs $\times 400$.

dent manner (Fig. 1). Significant inhibition of adhesion was observed at BSA concentrations over $0.5 \%$ or $1 \%$ depending on cell type. To test whether the effect of albu$\min$ is specific to hematopoietic cells, we investigated the effects of FBS and albumin on cell adhesion in rat C6 glioma and HCT116 human colon cancer cells. As shown in Fig. 2, we found that adhesion of $\mathrm{C} 6$ cells was not influenced by FBS deprivation. BSA disrupted cell adhesion of C6 cells in a concentration-dependent manner (Fig. 2). However, higher BSA concentration (5\%) was necessary to inhibit the cell adhesion of C6 glioma cells than that required to inhibit U-937 monocytes. Also, severe aggregation was observed in C6 glioma cells (Fig. 2). Similar results of cell adhesion in C6 glioma cells were also observed in HCT116 colon cancer cells (data not shown).
This implies that high concentrations of BSA blocked interaction between C6 gloima cells and ECM (extracellular matrix); therefore cells started to aggregate because integrin-mediated cell adhesion to tissue culture plates was not possible due to BSA blocking. The inhibitory effect of BSA, however, was more clearly observed at very low concentrations in hematopoietic cells as shown in Fig. 2 (Fig. 2). Therefore, the inhibitory effect of BSA on cell adhesion is considered to be specific to hematopoietic cells, because the physiological concentration of albumin in plasma is $0.6 \mathrm{mM}$ and adherent cells are rarely in the blood stream (Quinlan et al., 2005).

\section{Effect of antioxidants on cell adhesion}

Next, we tested the effects of various antioxidants on
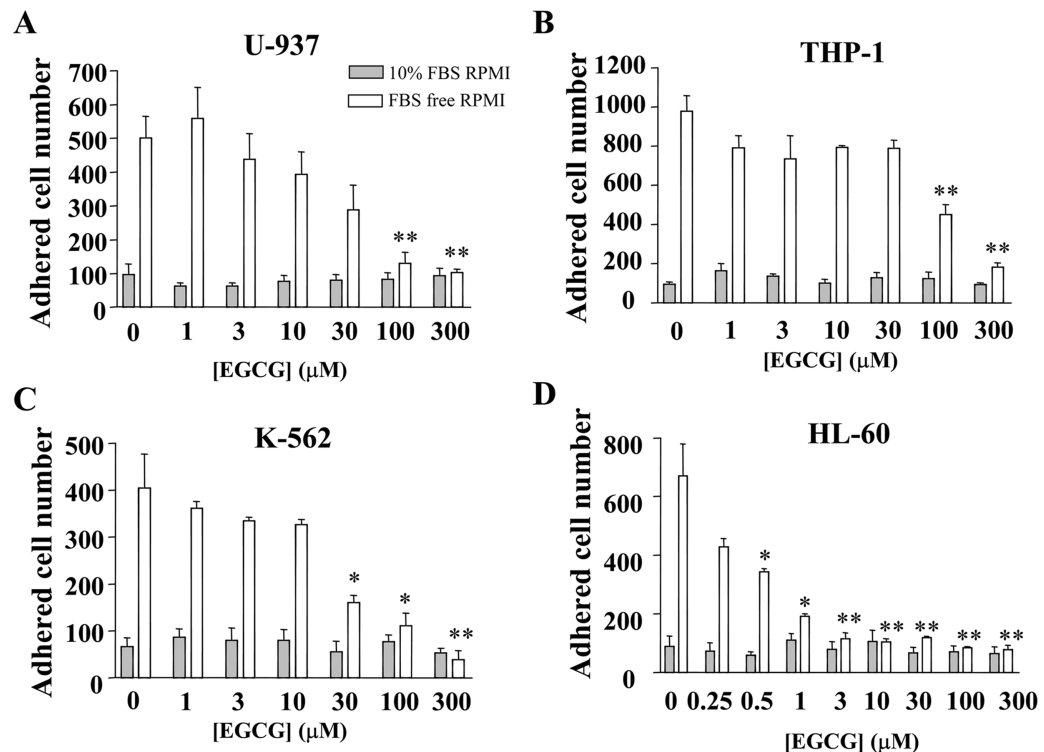

Fig. 3. Effect of EGCG on cell adhesion of hematopoietic cells EGCG concentration-dependence of cell adhesion in U-937 (A), THP-1 (B), K-562 (C), and HL-60 cells (D). Cells were incubated for $30 \mathrm{~min}$ in tissue culture dishes with 10\% FBS (filled column) or without FBS (open column) in the presence of various concentrations of EGCG, and the number of adherent cells was calculated. Statistical significance: ${ }^{*} P<0.05$, ${ }^{* *} P<0.01$ vs. control without FBS and EGCG. 
A

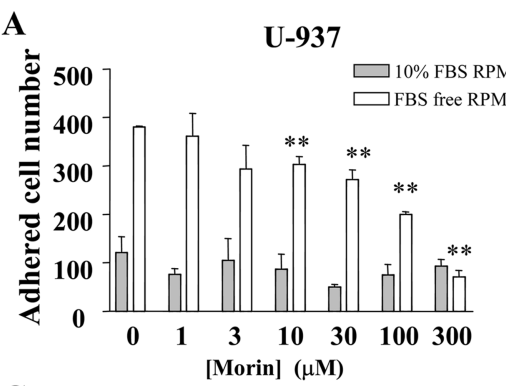

C

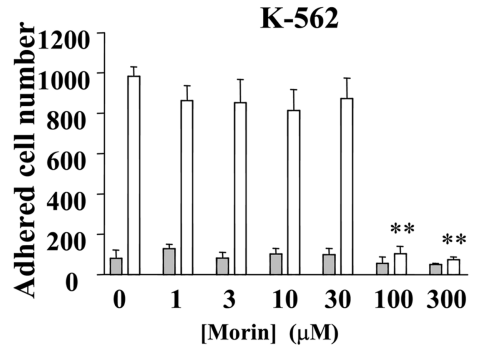

B

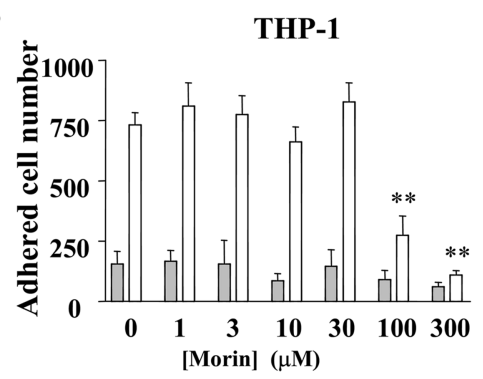

D

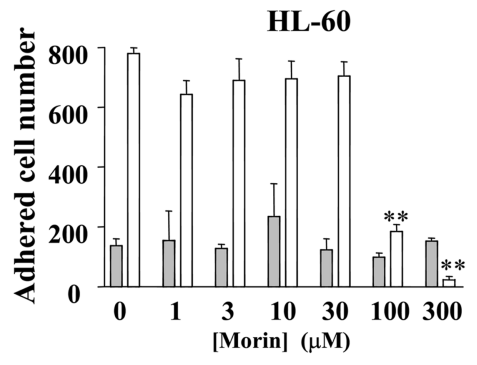

Fig. 4. Effect of morin on cell adhesion of hematopoietic cells Morin concentration-dependence of cell adhesion in U-937 (A), THP-1 (B), K-562 (C), and HL-60 cells (D). Cells were incubated for $30 \mathrm{~min}$ in tissue culture dishes with 10\% FBS (filled column) or without FBS (open column) in the presence of various concentrations of morin, and the number of adherent cells was calculated. Statistical significance: ${ }^{*} P<0.05,{ }^{* *} P<0.01$ vs. control without FBS and morin.

cell adhesion in the four hematopoietic cell lines, because oxidative signaling has been implicated in inflammationmediated cell adhesion (Chen et al., 2006; Sen and Roy, 2001). As shown in Fig. 3, EGCG, a polyphenolic antioxidant catechin from green tea, significantly inhibited the cell adhesion in a concentration-dependent manner in all tested cell lines. The effect was most potent in HL-60 leukemic cells, where complete inhibition was observed at 3 $\mu$ M EGCG. However, for U-937, THP-1, and K-562 cells, $100 \sim 300 \mu \mathrm{M}$ EGCG was necessary for complete inhibition. Morin, a flavone antioxidant from the Moraceae family, which includes mulberry figs, also inhibited the cell
A

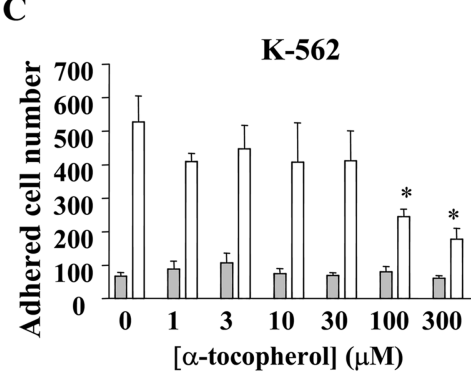

B

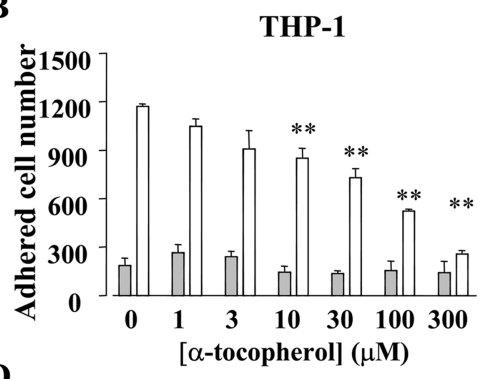

D

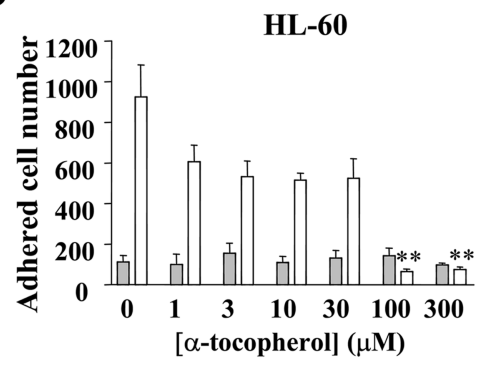

Fig. 5. Effect of $\alpha$-tocopherol on cell adhesion of hematopoietic cells $\alpha$-Tocopherol concentration-dependence of cell adhesion in $U$ 937 (A), THP-1 (B), K-562 (C), and HL-60 cells (D). Cells were incubated for 30 min in tissue culture dishes with 10\% FBS (filled column) or without FBS (open column) in the presence of various concentrations of $\alpha$-tocopherol, and the number of adherent cells was calculated. Statistical significance: * $P<0.05,{ }^{* *} P<0.01$ vs. control without FBS and without $\alpha$-tocopherol. 
adhesion of all cell types (Fig. 4); U-937 monocytes were more sensitive to morin than the other cell lines. In order to confirm whether oxidative signaling is involved in cell adhesion, we tested the effect of $\alpha$-tocopherol, the lipidsoluble antioxidant vitamin, in cell adhesion. As shown in Fig. 5, $\alpha$-tocopherol significantly inhibited the adhesion of the four tested cell lines in a concentration-dependent manner.

\section{DISCUSSION}

To expand on our previous findings, we tested four hematopoietic cells and found that, without serum, all cell types adhere to culture flasks and that the adhesion is inhibited by albumin. To generalize our previous findings: 1) Hematopoietic cells adhere to culture flasks in serumfree conditions, 2) Albumin in serum is the main inhibitory factor for maintaining the non-adherence of hematopoietic cells. Although in vivo experiments are necessary to support the physiological significance of our findings, it is obvious that albumin in serum blocks basal cell adhesion of hematopoietic cells in vitro. The cell adhesion of hematopoietic cells to culture flasks differed from that of C6 glioma cells to culture flasks. FBS deprivation did not influence the C6 glioma cell adhesion, in contrast to the dependence of hematopoietic cell adhesion on FBS deprivation. Furthermore, BSA did not influence C6 glioma cell adhesion at low concentrations ( 0.1 and $1 \%)$, excluding the non-specific blocking effect of BSA which caused the inhibition in hematopoietic cells. We tested the effects of various antioxidants and found that cell adhesion was abolished by treatment with antioxidants such as EGCG, morin, and $\alpha$-tocopherol. These three chemicals have different chemical structures, but have an antioxidant function in common. Furthermore, two other antioxidants, resveratrol and $\mathrm{N}$-acetyl-L-cysteine also showed inhibitory effects on cell adhesion in these four cell lines (data not shown). Therefore, oxidative signaling is thought to be important for the adhesion process. In the literature, some other mechanisms of action have been reported for those antioxidants. The action of $\alpha$-tocopherol is thought to be mediated by its antioxidant properties, mainly by protecting the polyunsaturated fatty acids in the plasma membrane and the membrane qualities (fluidity, phase separation, and lipid domains) (Traber and Atkinson, 2007), although an unknown target of $\alpha$-tocopherol has also been suggested (Azzi, 2007). In addition to its antioxidant function, morin has been shown to exhibit anti-inflammatory and antitumor activities through suppression of NF- $\mathrm{KB}$ activation (Manna et al., 2007). EGCG, a polyphenolic compound from green tea, has antioxidative, antithrombogenic, antiallergic, and anti-inflammatory properties. Its inhibitory action on cell adhesion has previously been studied in the prevention of atherosclerosis and cancer metastasis (Hung et al., 2005; Ludwig et al., 2004; Zhang et al., 2000). The interaction of EGCG with fibronectin and laminin was also suggested as a mechanism (Sazuka et al., 1998; Suzuki and Isemura, 2001). Binding of EGCG to CD11b, a member of $\alpha$-chain integrin in CD8 ${ }^{+} \mathrm{T}$ cells, was shown to attenuate adhesion and migration of $\mathrm{CD}^{+} \mathrm{T}$ cells (Kawai et al., 2004). In a later study, however, multiple mechanisms were suggested to explain the EGCGinduced inhibition of fibroblast adhesion and migration, including decreased expression and affinity of integrin a2 $\beta 1$, decrease of intracellular $\mathrm{H}_{2} \mathrm{O}_{2}$, focal adhesion kinase phosphorylation, actin cytoskeleton reorganization, and inhibition of matrix metalloproteinase activity and expression (Hung et al., 2005). The differential sensitivity of these cell lines to each antioxidant might imply that another mode of action is in effect for these three antioxidants, other than the antioxidant function, such as in the multiple mechanisms of action of EGCG (Hung et al., 2005). However, the antioxidative function of these three chemicals is thought to be the main cause of the inhibition of cell adhesion. Inappropriate or abnormal sequestration of leukocytes at specific sites is a central component in the development of a variety of autoimmune diseases and pathologic inflammatory disorders (Collins et al., 1994; Dailey, 1998).

Cell adhesion molecule expression and adhesive properties of cells are greatly modified by several conditions involving redox imbalances (Sen and Roy, 2001). Oxidants such as $\mathrm{H}_{2} \mathrm{O}_{2}$ are known to induce adherence of leukocytes to endothelial cells (Roy et al., 1999; Sellak et al., 1994). Several antioxidants down-regulate inducible cell adhesion molecule expression as well as cell-cell adhesion (Sen and Roy, 2001). Agonist (interleukin-1, thrombin, or PMA)-induced monocytic adhesion to HUVEC, but not basal adhesion, was down-regulated by $\alpha$-tocopherol (Faruqi et al., 1994). Quercetine, a potent flavonoid antioxidant, suppressed agonist-induced ICAM-1 protein and gene expression (Kobuchi et al., 1999). Inflammatory cytokine-stimulated endothelial adhesion to human monocytic cells could be suppressed by drugs with antioxidant activity that attenuated intracellular ROS formation, redox-sensitive transcription factor activation, and VCAM-1 and ICAM-1 expression (Chen et al., 2006). Another interpretation of our results is that the cells were not activated by any stimulants. Adhesion and migration of hematopoietic cells to endothelial cells are evoked by 
stimuli such as inflammatory cytokines. However, in our experiment, the withdrawal of serum made cells adhere and the presence of albumin inhibited cell adhesion. Therefore, our results may imply the significance of hematopoietic cell basal adhesion and its modulation by albumin and oxidative balance in plasma. In summary, in the present study, we found that albumin-induced inhibition of cell adhesion is common in hematopoietic cells and that antioxidants inhibit the basal cell adhesion of hematopoietic cells in vitro. These results imply that albumin and the redox status in plasma may modulate basal adhesion of hematopoietic cells in vivo.

\section{ACKNOWLEDGEMENT}

This work was supported by the Grand-in-aid for 2007 Korea-Japan Joint Research Project from Korea Science and Engineering Foundation (F01-2007-000-10005-0).

\section{REFERENCES}

Azzi, A. (2007). Molecular mechanism of alpha-tocopherol action. Free Radic. Biol. Med. 43, 16-21.

Campanero, M. R., Sanchez-Mateos, P., del Pozo, M. A. and Sanchez-Madrid, F. (1994). ICAM-3 regulates lymphocyte morphology and integrin-mediated $\mathrm{T}$ cell interaction with endothelial cell and extracellular matrix ligands. J. Cell Biol. 127, 867-878.

Chen, Y. H., Lin, S. J., Chen, Y. L., Liu, P. L. and Chen, J. W. (2006). Anti-inflammatory effects of different drugs/agents with antioxidant property on endothelial expression of adhesion molecules. Cardiovasc. Hematol. Disord. Drug Targets 6, 279-304.

Collins, T. L., Kassner, P. D., Bierer, B. E. and Burakoff, S. J. (1994). Adhesion receptors in lymphocyte activation. Curr. Opin. Immunol. 6, 385-393.

Dailey, M. O. (1998). Expression of T lymphocyte adhesion molecules: regulation during antigen-induced $T$ cell activation and differentiation. Crit. Rev. Immunol. 18, 153-184.

Dustin, M. L. and de Fougerolles, A. R. (2001). Reprogramming $T$ cells: the role of extracellular matrix in coordination of $T$ cell activation and migration. Curr. Opin. Immunol. 13, 286-290.

Faruqi, R., de la Motte, C. and DiCorleto, P. E. (1994). Alphatocopherol inhibits agonist-induced monocytic cell adhesion to cultured human endothelial cells. J. Clin. Invest. 94, 592-600.

Hung, C. F., Huang, T. F., Chiang, H. S. and Wu, W. B. (2005). (-)-Epigallocatechin-3-gallate, a polyphenolic compound from green tea, inhibits fibroblast adhesion and migration through multiple mechanisms. J. Cell Biochem. 96, 183-197.

Kawai, K., Tsuno, N. H., Kitayama, J., Okaji, Y., Yazawa, K.,
Asakage, M., Hori, N., Watanabe, T., Takahashi, K. and Nagawa, H. (2004). Epigallocatechin gallate attenuates adhesion and migration of CD8+ T cells by binding to CD11b. $J$. Allergy Clin. Immunol. 113, 1211-1217.

Kim, Y. L., Im, Y. J., Lee, Y. K., Ha, N. C., Bae, Y. S., Lim, S. M., Okajima, F. and Im, D. S. (2006). Albumin functions as an inhibitor of T cell adhesion in vitro. Biochem. Biophys. Res. Commun. 351, 953-957.

Kobuchi, H., Roy, S., Sen, C. K., Nguyen, H. G. and Packer, L. (1999). Quercetin inhibits inducible ICAM-1 expression in human endothelial cells through the JNK pathway. Am. J. Physiol. 277, C403-411.

Ludwig, A., Lorenz, M., Grimbo, N., Steinle, F., Meiners, S., Bartsch, C., Stangl, K., Baumann, G. and Stangl, V. (2004). The tea flavonoid epigallocatechin-3-gallate reduces cytokineinduced VCAM-1 expression and monocyte adhesion to endothelial cells. Biochem. Biophys. Res. Commun. 316, 659-665.

Manna, S. K., Aggarwal, R. S., Sethi, G., Aggarwal, B. B. and Ramesh, G. T. (2007). Morin (3,5,7,2',4'-Pentahydroxyflavone) abolishes nuclear factor-kappaB activation induced by various carcinogens and inflammatory stimuli, leading to suppression of nuclear factor-kappaB-regulated gene expression and up-regulation of apoptosis. Clin. Cancer Res. 13, 22902297.

Quinlan, G. J., Martin, G. S. and Evans, T. W. (2005). Albumin: biochemical properties and therapeutic potential. Hepatology 41, 1211-1219.

Roy, S., Sen, C. K. and Packer, L. (1999). Determination of cellcell adhesion in response to oxidants and antioxidants. Methods Enzymol. 300, 395-401.

Sazuka, M., Isemura, M. and Isemura, S. (1998). Interaction between the carboxyl-terminal heparin-binding domain of fibronectin and (-)-epigallocatechin gallate. Biosci. Biotechnol. Biochem. 62, 1031-1032.

Sellak, H., Franzini, E., Hakim, J. and Pasquier, C. (1994). Reactive oxygen species rapidly increase endothelial ICAM-1 ability to bind neutrophils without detectable upregulation. Blood 83, 2669-2677.

Sen, C. K. and Roy, S. (2001). Antioxidant regulation of cell adhesion. Med. Sci. Sports Exerc. 33, 377-381.

Suzuki, Y. and Isemura, M. (2001). Inhibitory effect of epigallocatechin gallate on adhesion of murine melanoma cells to laminin. Cancer Lett. 173, 15-20.

Traber, M. G. and Atkinson, J. (2007). Vitamin E, antioxidant and nothing more. Free Radic. Biol. Med. 43, 4-15.

Tseng, S. Y. and Dustin, M. L. (2002). T-cell activation: a multidimensional signaling network. Curr. Opin. Cell Biol. 14, 575580.

Zhang, G., Miura, Y. and Yagasaki, K. (2000). Suppression of adhesion and invasion of hepatoma cells in culture by tea compounds through antioxidative activity. Cancer Lett. 159, 169-173. 